\title{
Analytical recursive method to ascertain multisite entanglement in doped quantum spin ladders
}

\author{
Sudipto Singha Roy ${ }^{1,2}$, Himadri Shekhar Dhar ${ }^{1,2,3}$, Debraj Rakshit ${ }^{1,2,4}$, Aditi Sen(De $)^{1,2}$, and Ujjwal Sen ${ }^{1,2}$ \\ ${ }^{1}$ Harish-Chandra Research Institute, Chhatnag Road, Jhunsi, Allahabad 211 019, India \\ ${ }^{2}$ Homi Bhabha National Institute, Training School Complex, Anushakti Nagar, Mumbai 400 085, India \\ ${ }^{3}$ Institute for Theoretical Physics, Vienna University of Technology, \\ Wiedner Hauptstrae 8-10/136, A-1040 Vienna, Austria \\ ${ }^{4}$ Institute of Physics, Polish Academy of Sciences, \\ Aleja Lotników 32/46, PL-02668 Warsaw, Poland
}

(Dated: October 8, 2018)

\begin{abstract}
We formulate an analytical recursive method to generate the wave function of doped short-range resonating valence bond (RVB) states as a tool to efficiently estimate multisite entanglement as well as other physical quantities in doped quantum spin ladders. We prove that doped RVB ladder states are always genuine multipartite entangled. Importantly, our results show that within specific doping concentration and model parameter regimes, the doped RVB state essentially characterizes the trends of genuine multiparty entanglement in the exact ground states of the Hubbard model with large onsite interactions, in the limit which yields the $t$ - $J$ Hamiltonian.
\end{abstract}

\section{INTRODUCTION}

One of the best known and simplest theoretical frameworks for investigating strongly-correlated doped quantum spin ladders is the $t-J$ model, which is obtained in the limit of large on-site interaction from the Hubbard model $^{1-4}$. At half-filling, without doping, the system reduces to a Heisenberg ladder with a spin liquid ground state $(\mathrm{GS})^{5}$. Upon doping the spin ladder, studies based on mean-field theory using Gutzwiller renormalization show that the spin gap is persistent ${ }^{6}$ which is a tell-tale sign of strong superconducting fluctuations ${ }^{5-7}$. The $t-J$ model, under finite doping, exhibits a rich phase diagram, which has been extensively studied for low-dimensional antiferromagnets $(\mathrm{AFM})^{8-11}$. In particular, in $1 \mathrm{D}$ and ladder configurations, the system possess exotic correlation properties that are characterized by the Luttinger liquid theory ${ }^{12}$, as confirmed using exact diagonalization calculations, and exhibits a rich superconducting phase for a specific range of values of $J / t$ and electron density, $n_{e l}{ }^{8-11,13,14}$. Moreover, the superconducting states of the quantum spin ladder can be represented using the shortrange resonating valence bond $(\mathrm{RVB})$ ansatz ${ }^{15,16}$ which were introduced to describe Mott-insulators.

An important yet demanding task in the study of doped quantum spin ladders is to characterize how quantum correlations, in particular multiparty entanglement, are distributed among the subparts of these strongly correlated systems. This is motivated, on one hand, by the fact that study of multisite physical quantities in many-body quantum systems often provide deeper insights into the cooperative phenomena they exhibit ${ }^{17,18}$. On the other hand, such investigation can play an important role for implementation of quantum information processing tasks in the laboratory. However, estimation of the same remains a challenging task, primarily due to the exponential growth of the Hilbert space with increasing system size. This is especially true if we try to obtain analytical expressions or bounds of multisite physical properties such as entanglement. Therefore, obtaining a general method to characterize entanglement in multipartite states is crucial to investigate physical phenomena of a complex system.

In this work, we consider short-range doped resonating valence bond (RVB) states, and for finite values of the electron density $\left(n_{e l}\right)$, using the symmetry properties of the RVB state ${ }^{19-21}$, we prove that the doped RVB ladder is always genuinely multipartite entangled. To quantify the genuine multiparty entanglement in large spin ladders, we introduce an analytical recursion method to build the doped RVB state. The novelty of this recursion method stems from the fact that in a large spin network with arbitrary electron density $\left(n_{e l}\right)$, one can analytically compute the reduced density matrices of the superposition state, thus allowing an exact estimation of the genuine multiparty entanglement using the generalized geometric measure $(\mathcal{G})^{22}$ (cf. Refs. ${ }^{23,24}$ ). Using the proposed recursion method, we observe that in the thermodynamic limit, $\mathcal{G}$ increases with $n_{e l}$, reaching a maximum at $n_{c} \approx 0.56$, before decreasing for higher $n_{e l}$ $\left(\mathrm{cf}^{25}\right)$. Interestingly, we further show that the qualitative multipartite features of doped RVB states are closely mimicked by ground states (GSs) of doped $t$ - $J$ ladders obtained through exact diagonalization for moderate-sized lattices. In particular, we present a representative case with $J / t \approx 0.6$, where we observe that genuine multiparty entanglement of the GS of the $t-J$ ladder emulates the same of the doped RVB state. The maximum $\mathcal{G}$ occurs at $n_{c} \approx 0.65$, close to that obtained using the doped RVB ansatz. The discrepancy in the values of electron densities need to account for finite size effect. Hence, using the analytical recursion method, one can show that within the considered parameter range, the trend of genuine multipartite entanglement of the former state qualitatively matches with that of the GS of the latter model. We note that although we use the recursion method to study multipartite entanglement, the method can also be employed to investigate other properties like single-site, 
two-site physical quantities of the doped RVB ladders for systems with an arbitrary number of sites. In our calculations, we have considered up to 300 sites, and though a higher number of sites are accessible through our method, the physical quantities of interest converge much earlier.

The paper is organized as follows. In Sec. II, we present the recursion method that generates the doped RVB state corresponding to arbitrary electron density. Thereafter, in Sec III, we propose the recursion relation for density matrices of multiple sites, considering both periodic as well as open boundary conditions. In Sec. IV, we provide analytical results on genuine multipartite entanglement in the doped RVB state. We also discuss a measure of genuine multipartite entanglement called the generalized geometric measure $(\mathcal{G})$ in Sec.V. In Sec. VI, we introduce the $t$ - $J$ model and subsequently compare the behavior of $\mathcal{G}$ obtained using the doped RVB state ansatz to that obtained via exact diagonalization of the $t-J$ model. We present a brief conclusion in Sec. VII.

\section{RECURSION METHOD TO GENERATE DOPED RVB STATES}

We begin by considering the doped RVB state containing $2 N$ lattice sites, on a ladder configuration, with $2 k$ spin- $1 / 2$ particles and $2(N-k)$ holes or vacant lattice sites, expressed using a bipartite lattice, consisting of sublattices $A$ and $B$. The corresponding (unnormalized) wavefunction, with electron density $n_{e l}=k / N$, is given by

$$
\begin{aligned}
|\Psi\rangle_{k, N-k}= & \sum_{i} r_{i}\left|\left(a_{n_{1}} b_{n_{1}}\right)\left(a_{n_{2}} b_{n_{2}}\right) \cdots\left(a_{n_{k}} b_{n_{k}}\right)\right\rangle_{i} \\
& \otimes\left|h_{m_{2 k+1}} h_{m_{2 k+2}} \cdots h_{m_{2 N}}\right\rangle_{i}
\end{aligned}
$$

where $\left|\left(a_{n_{j}} b_{n_{j}}\right)\right\rangle=\frac{1}{\sqrt{2}}(|01\rangle-|10\rangle)_{n_{j}}$ represents a dimer, with $a_{j} \in A$ and $b_{j} \in B . \quad\left|\left\{\left(a_{n_{j}} b_{n_{j}}\right)\right\}\right\rangle_{i}$ represents a complete dimer covering at occupied sites $n_{j}$. The holes, $\left|h_{m_{j}}\right\rangle=|2\rangle_{m_{j}}$, are at sites $m_{j}$, such that $\sum_{j=1}^{k} 2 n_{j}+$ $\sum_{j=2 k+1}^{2 N} m_{j}=2 N . r_{i}=1, \forall i$.

In general, in considering the RVB ansatz for the ground state of a moderate-sized doped quantum spin ladder, as described in Eq. (1), the number of dimer coverings in the state increases exponentially with the increase of the electron density ${ }^{26}$. For example, in a small spin ladder with 5 spins on each leg, the number of dimer coverings at electron density $n_{e l}=0.33$ 94, and at $n_{e l}=0.66$, it is equal to 294 . Hence, even for small ladders, a direct construction of the RVB ground state is computationally expensive. Moreover, the Hilbert space also increases rapidly with increase in the number of spins. This makes the analytical recursion method proposed for studying physical properties of doped RVB states on large quantum spin ladders, a very important part of our results. We recursively ${ }^{27-30}$ construct the state $|\psi\rangle_{k, N-k}$, defined in Eq. (1) and generate its reduced states. Though earlier attempts have been made to obtain recursion relations for physical observables such as the ground state energy ${ }^{30}$, the novelty of our approach lies in the fact that the proposed method recursively constructs the reduced density matrices of the doped RVB state, which allows us to study quantum and classical properties, in particular, multipartite entanglement, which in turn are used to characterize the system.

In order to generate the analytical recursion method, let us begin with an open $2 N$-site ladder lattice with all vacant sites (holes), which is successively filled with dimers. We use the notation, $|N-k, k\rangle$ to denote the $N$-rung ladder, $|\psi\rangle_{k, N-k}$, containing $2 k$ spins filled with dimers and $2(N-k)$ holes. The state $|N-k, k\rangle$ is achieved by successively filling $k$ dimers in the $|N, 0\rangle$ state, i.e., $|N, 0\rangle \stackrel{k}{\rightarrow}|N-k, k\rangle$. As an example, consider an initial configuration with 8 site RVB ladder, doped with 4 holes. Now the state $|2,2\rangle$ mentioned above, can be generated in the following way:

$$
|4,0\rangle \stackrel{k=1}{\longrightarrow}|3,1\rangle \stackrel{k=1}{\longrightarrow}|2,2\rangle,
$$

where $|4,0\rangle$ is the initial lattice with all holes, and $|2,2\rangle$ is the final state, for an 8 site RVB ladder, with 2 dimers and 2 pairs of holes.

For an analytical method which allows us to build the superpositions in an arbitrary $|N-k, k\rangle$, we propose the generator

$$
\begin{aligned}
& |N-k, k\rangle=\mathcal{U}^{\otimes k^{\prime}=1}|N-k+1, k-1\rangle \\
+ & |N-k-1,0\rangle\left|\chi_{k+1}\right\rangle+|N-k-2,0\rangle\left|\chi_{k+1}\right\rangle|1,0\rangle,
\end{aligned}
$$

where $\mathcal{U}^{\otimes k^{\prime}}$ is the operator to add $k^{\prime}$ dimers. The methodology to derive the above recursion relation and the description of $\left|\chi_{k+1}\right\rangle$ are given below.

To facilitate our calculations, we divide the $2 N$ ladder lattice into specific regions that can be filled with dimers. We start by splitting the initial state $|N, 0\rangle$ into two regions, denoted by left $(L)$ and right $(R)$ block, such that

$$
|N, 0\rangle=|N-2,0\rangle_{L} \otimes|2,0\rangle_{R} .
$$

This is explicitly shown in Fig. 1. An important region is the junction $(J)$ block between $L$ and $R$ blocks, which is shown in Fig. 1 using a black-dotted square. The blocks, excluding overlapping region, can be written as:

$$
|N-3,0\rangle_{L^{\prime}} \otimes|2,0\rangle_{J} \otimes|1,0\rangle_{R^{\prime}},
$$

where $L^{\prime}\left(R^{\prime}\right)$ implies the region $L-L \cap J(R-R \cap J)$.

Now starting from an initial configuration $|N, 0\rangle$, our aim is to reach the final state $|N-k, k\rangle$ by systematically introducing $k$ numbers of dimers in the different blocks of the lattice. The first dimer is introduced in the initial hole configuration though the following possible ways:

1. Update the left block: In this step, a dimer is introduced into the $L$ block and the updated state is

$$
|N-2,0\rangle_{L}|2,0\rangle_{R} \stackrel{k=1}{\longrightarrow}|N-3,1\rangle_{L}|2,0\rangle_{R} .
$$



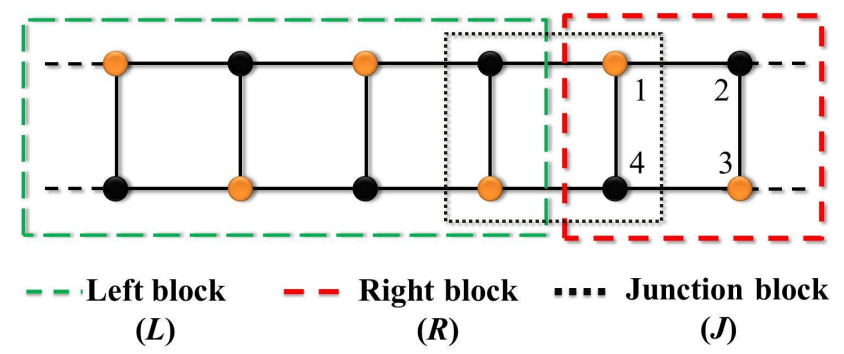

FIG. 1. Schematic diagram of the blocks $L, R$, and $J$ in the spin lattice. To compute $\mathcal{G}$, we obtain the reduced density matrix $\left(\rho_{\text {red }}\right)$ corresponding to the sites $1-4$ in the $R$ block. The rest of the lattice is traced out. Numerical studies show that the reduced state $\rho_{\text {red }}$ is sufficient to compute $\mathcal{G}$ in doped RVB states.

a)

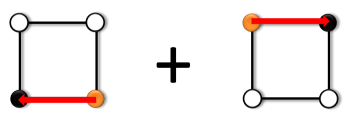

$\left|\chi_{2}\right\rangle$

c)

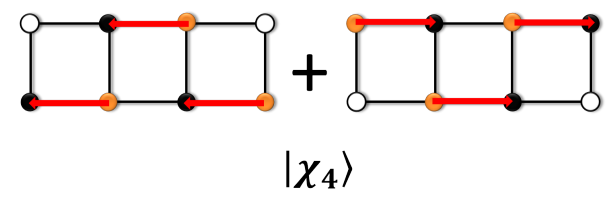

d)

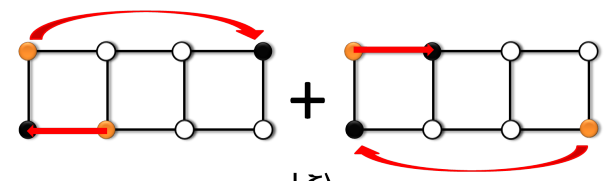

$|\xi\rangle$

e)

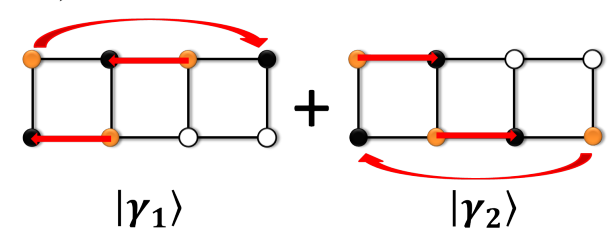

FIG. 2. Schematic diagram of the states: a) $\left|\chi_{2}\right\rangle$, b) $\left|\chi_{3}\right\rangle$, c) $\left|\chi_{3}\right\rangle$, and the periodic terms: d) $|\xi\rangle$, and e) $\left|\gamma_{1}\right\rangle$ and $\left|\gamma_{2}\right\rangle$, used in the recursion relations.

2. Update the right block: Similarly, in the next step, a dimer is injected into the $R$ block. The updated state looks like

$$
|N-2,0\rangle_{L}|2,0\rangle_{R} \stackrel{k=1}{\longrightarrow}|N-2,0\rangle_{L}|1,1\rangle_{R}
$$

3. Update of the junction block: In this step a dimer is introduced in the junction of the $L$ and $R$ blocks, i.e., the $J$ block. The updated state turns out to be

$$
|N-2,0\rangle_{L}|2,0\rangle_{R} \stackrel{k=1}{\longrightarrow}|N-1,0\rangle\left|\chi_{2}\right\rangle|1,0\rangle,
$$

where the state $\left|\chi_{2}\right\rangle$ is depicted in Fig. 2(a). Now combining the above three steps, the final state after introduction of a single dimer in the lattice is given by

$$
\begin{aligned}
|N, 0\rangle \stackrel{k=1}{\longrightarrow}|N-1,1\rangle \\
\equiv|N-3,1\rangle_{L}|2,0\rangle_{R}+|N-2,0\rangle_{L}|1,1\rangle_{R} \\
+|N-1,0\rangle\left|\chi_{2}\right\rangle|1,1\rangle .
\end{aligned}
$$

For example, consider the initial state $|4,0\rangle$ in Eq. (2). We have, $|4,0\rangle=|2,0\rangle_{L}|2,0\rangle_{R}$. Then the state, after introduction of one dimer, would be (see Fig. 3 for an illustration of the three update paths)

$$
\begin{aligned}
|4,0\rangle \stackrel{k=1}{\longrightarrow}|3,1\rangle=|1,1\rangle|2,0\rangle+|2,0\rangle|1,1\rangle \\
+|1,0\rangle\left|\chi_{2}\right\rangle|1,0\rangle,
\end{aligned}
$$

where the first two terms are the contributions from the blocks $L$ and $R$, and the third term comes from the update of the junction, $J$. Now after completion of the first step, we need to introduce one more dimer into the present configuration in order to continue the iteration process. It can be done following a path similar to the one described above, i.e., a direct update of the $L$ and $R$ blocks, which is basically updating all the terms of the state by introducing dimers into the left and right blocks, and an update, which consists of injecting a dimer at the junction block. The above scheme can be repeated $k$ times so that the final state contains $k$ dimers and $2(N-k)$ holes in the lattice. In general, by updating the $L, J$, and $R$ blocks, with $k^{\prime}=1$ singlets, we obtain the recursive generator expressed in Eq. (2). As mentioned before, here $\mathcal{U}^{\otimes k^{\prime}}$ is the direct update operator to inject $k^{\prime}$ dimers in the $L$ and $R$ blocks of the state, $|N-k+1, k-1\rangle$. Subsequently, the second and the third terms in Eq. (2) correspond to the indirect update of the $J$ block. For example, the first two terms in Eq. (8), |1, 1 $|2,0\rangle$ and $|2,0\rangle|1,1\rangle$, is generated from the direct update of the state $|4,0\rangle$ and the third term $|1,0\rangle\left|\chi_{2}\right\rangle|1,0\rangle$ emerges from the indirect update of the junction sites. Note that, there may arise similar terms due to the update process of the $L, R$, and the $J$ block. In those cases we need to carefully include such terms only once in the recursion, so that overcounting of the terms can be avoided. In Eq. (2), we note that the term $\left|\chi_{k+1}\right\rangle$ can be generated recursively from $\left|\chi_{k}\right\rangle$ by introducing an additional rung to the left and assigning a dimer along the horizontal direction, as demonstrated in Fig. 2, for $\left|\chi_{2}\right\rangle,\left|\chi_{3}\right\rangle$ and $\left|\chi_{4}\right\rangle$.

In the succeeding section, we present a detailed discussion on how reduced density matrices for a block of lattice sites can be obtained from the recursion method. For the purposes of our study, a block of four sites, in two nearest neighbor (NN) rungs of the ladder, is sufficient. 


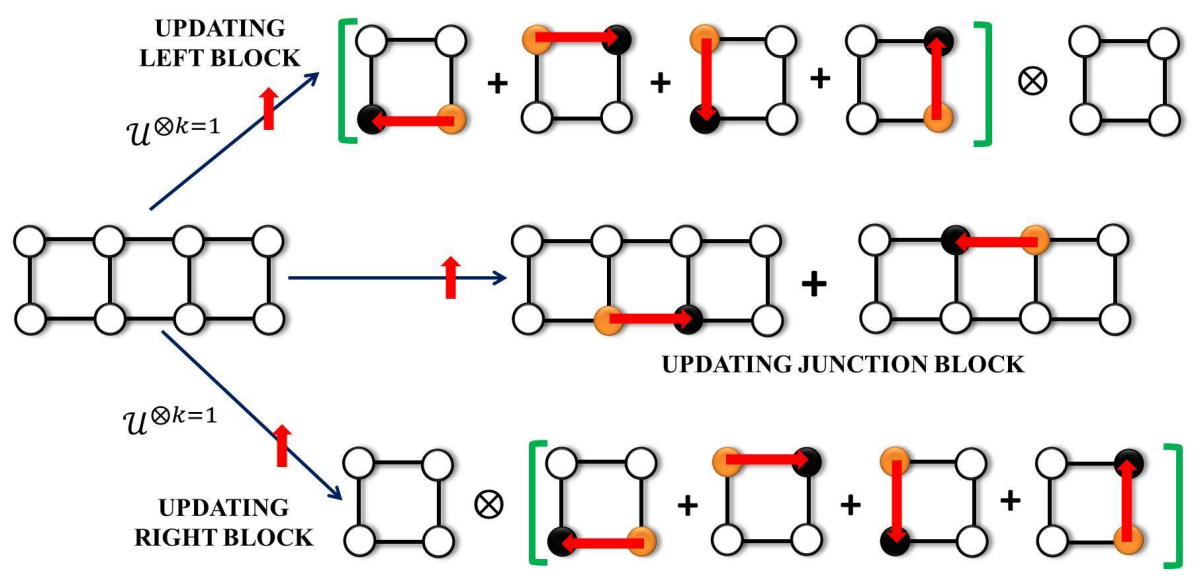

FIG. 3. Schematic diagram of the scheme to build a doped-RVB state from a lattice filled with holes, for an 8 site RVB ladder, as given by the process: $|4,0\rangle \stackrel{k=1}{\longrightarrow}|3,1\rangle$, described in Eq. (2).

\section{RECURSION RELATION FOR REDUCED DENSITY MATRICES}

In order to calculate the $\mathcal{G}$ of the doped RVB state, we required to derive expressions for the reduced density matrices, using the generator expressed in Eq. (2). Let us consider the cases for open and periodic ladders separately. a) Open ladder: The primary method to build the recursive relations is to divide the lattice into blocks and junctions. The advantage lies in the fact that these blocks do not overlap, and hence can be independently traced to obtain $\rho_{\text {red }}$ needed to calculate the $\mathcal{G}$. Hence, from the non-periodic ladder state, $|N-k, k\rangle$, by tracing all sites apart from rungs $m-1$ and $m$, we get the reduced state, $\rho_{\text {red }}^{\mathcal{N} \mathcal{P}}$, of 4 -sites , given by

$$
\begin{aligned}
\boldsymbol{\rho}_{\text {red }}^{\mathcal{N P}} & =\sum_{i=0}^{2} \mathcal{Z}_{(k-i)}^{(\mathcal{S}-1+i)}|2-i, i\rangle\left\langle 2-i, i\left|+\sum_{k_{1}=2}^{k+1} \mathcal{Z}_{\left(k-k_{1}+1\right)}^{(\mathcal{S}-1)} \operatorname{tr}\left(\left|\chi_{k_{1}}\right\rangle\left\langle\chi_{k_{1}}\right|\right)\right| 1^{\prime}\right\rangle\left\langle 1^{\prime}\left|+\sum_{k_{2}=2}^{k} \mathcal{Z}_{\left(k-k_{2}\right)}^{(\mathcal{S})} \operatorname{tr}\left(\left|\chi_{k_{2}}\right\rangle\left\langle\chi_{k_{2}}\right|\right)\right| 1\right\rangle\langle 1| \\
& +\sum_{i=0}^{1} \mathcal{Z}_{(k-2-i)}^{(\mathcal{S}+i)} \operatorname{tr}(|\overline{2}\rangle\langle\overline{2}|)|1-i, i\rangle\langle 1-i, i|+\sum_{k_{3}=3}^{k+1} \mathcal{Z}_{\left(k-k_{3}+1\right)}^{(\mathcal{S})}\left(\operatorname{tr}\left(\left|\chi_{k_{3}}\right\rangle\left\langle\chi_{k_{3}}\right|\right)\right)+\sum_{k_{4}=2}^{k} \mathcal{Z}_{\left(k-k_{4}\right)}^{(\mathcal{S})}\left(\operatorname{tr}\left(\left|\chi_{k_{4}+1}\right\rangle\left\langle\chi_{k_{4}}\right|\langle 1|\right)\right. \\
& + \text { h.c. })+\sum_{\substack{i=1, j=k-2-i}} \mathcal{Z}_{(k-2-i-j)}^{(\mathcal{S}+i)}(1 / 2)^{j+1}(|1\rangle|1-j, j\rangle\langle 1-j, j+1|+\text { h.c. }), \text { where } \mathcal{S}=N-k-1, \text { and }
\end{aligned}
$$

$\mathcal{Z}_{k}^{N-k}=\langle N-k, k \mid N-k, k\rangle$, and $|\overline{2}\rangle=|0,2\rangle-|0,1\rangle|1,0\rangle$. Numerical studies for a moderate $N$, suggest that obtaining the reduced state of a square block of 4 sites for large ladders, which is symmetric for the ladder, is sufficient for the computation of $\mathcal{G}$. Hence, we use the recursion method to obtain the 4 -site reduced state $\left(\rho_{\text {red }}\right)$ at rungs $m-1$ and $m$.

The main advantage in formulating the recursion relation for the entire state, as expressed in Eq. (2), can be seen when one needs to obtain the reduced density matrix, $\rho_{\text {red }}$. This is because the terms which correspond to the blocks $R$ and $L$ are mutually orthogonal to those belong to the junction block $J$. As a result, in the expression for $\rho_{\text {red }}$, one would never get any contribution from the terms that emanate from $|\bullet\rangle_{L(R)}\left\langle\left.\bullet\right|_{J}\right.$. Now if one starts from the $L$ and $R$ blocks coverings and traces out all but the sites of last two rungs (sites (1-4) in Fig. 1), then there would be the following three possibilities,

i) The reduced block contains holes only. ii) The reduced block contains one singlet and one pair of hole. iii) The reduced block contains singlet coverings only.

The first term in Eq. (9),

$$
\sum_{i=0}^{2} \mathcal{Z}_{(k-i)}^{(\mathcal{S}-1+i)}|(2-i),(i)\rangle\langle(2-i),(i)|
$$

basically corresponds to the above possibilities. As an example, consider an initial eight-site doped RVB state which includes only one singlet and 3 hole-pairs. The contribution from the $L$ block and $R$ block would lead to the following terms in the expression of the doped 
RVB state, $|1,1\rangle_{L} \otimes|2,0\rangle_{R}+|2,0\rangle_{L} \otimes|1,1\rangle_{R}$. Therefore, the reduced state would contain following terms, $a_{1}|2,0\rangle\left\langle 2,0\left|+a_{2}\right| 1,1\right\rangle\langle 1,1|$, where $a_{1}=\mathcal{Z}_{1}^{1}$, and $a_{2}=$ $\mathcal{Z}_{0}^{2}$, which can be obtained from Eq. (9).

Subsequently, the junction $J$ would generate additional terms in the expression of the reduced state. As an example, first consider terms which has only one singlet at the junction block (see Fig. 2). Mathematically, those can be expressed as $|\bullet\rangle \otimes\left|\chi_{k}\right\rangle \otimes|\bullet\rangle$, where $\left|\chi_{2}\right\rangle$ and $\left|\chi_{3}\right\rangle$ are depicted in Fig. 2. Now the contributions from the overlap of those terms are given by second, third, and fourth terms in Eq. (9). Considering, once more, the previous example of an eight-site doped RVB state containing only one singlet, we can write the contributing term from the junction as $|1,0\rangle \otimes\left|\chi_{2}\right\rangle \otimes|1,0\rangle$. Hence after tracing out all but the sites those are at the last two-rungs, we get

$$
\begin{aligned}
\rho_{\text {red }}^{\mathcal{N} \mathcal{P}}= & a_{1}|2,0\rangle\left\langle 2,0\left|+a_{2}\right| 1,1\right\rangle\langle 1,1| \\
& +a_{3} \operatorname{tr}\left|\chi_{2}\right\rangle\left\langle\chi_{2}|\otimes| 1,0\right\rangle\langle 1,0|,
\end{aligned}
$$

where $a_{3}=\mathcal{Z}_{0}^{1}$ is again evaluated using Eq. (9). (a)

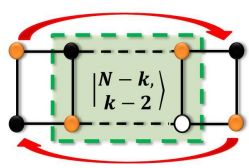

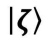

(b)

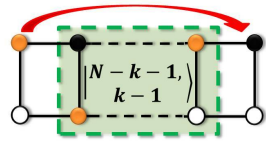

$\left|\eta_{1}\right\rangle$ (c)

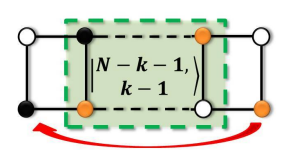

$\left|\eta_{2}\right\rangle$
FIG. 4. Schematic diagram of the blocks $L, R$, and $J$ in the spin lattice with periodic boundary condition.

Additionally, there may be terms which would contain two horizontal singlets at the junctions such as $|\bullet\rangle \otimes$ $|\overline{2}\rangle \otimes|\bullet\rangle$. Those would certainly have non-zero overlap with the $L$ and $R$ block terms. The fifth and sixth terms of Eq. (9) correspond to contribution from these terms. As an example, instead of inserting one singlet if we now introduce two singlets in the eight-site doped RVB state i.e. $|2,2\rangle$, we would have terms in the doped RVB state such as $|1,0\rangle \otimes|\overline{2}\rangle \otimes|1,0\rangle$. Hence the reduced density matrix would have following terms,

$$
a_{4} \operatorname{tr}|\overline{2}\rangle\langle\overline{2}|\otimes| 1,0\rangle\left\langle 1,0\left|+a_{5} / 2\right| 0,1\right\rangle|1,0\rangle\langle H|,
$$

where $a_{4}$ and $a_{5}$ are given by $\mathcal{Z}_{0}^{1}$ which can be obtained from Eq. (9), for $N=4$ and $k=2$.

b) Periodic ladder: Incorporation of periodic boundary condition, $N+1 \equiv N$, leads to additional terms in the state $|N-k, k\rangle$. Consequently, we find that the number of terms in the expression of the recursively generated reduced density matrix, $\rho_{\text {red }}^{\mathcal{P}}$, increases, due to overlap of different states. These extra terms in $\rho_{\text {red }}^{\mathcal{P}}$ can be redeemed by analyzing two separate situations, say $\mathcal{P}_{1}$ and $\mathcal{P}_{2}$, corresponding to different boundary terms:

I) $\mathcal{P}_{1}$ - the end sites of both the legs share a singlet (see Fig. 4(a)).

II) $\mathcal{P}_{2}-$ the end sites of only one of the leg share a singlet and the end site of the other leg contains holes (see Figs. 4(b) and (c))).

Therefore, in the expression of the reduced density matrix, there would be additional terms due to the overlap of the states in I with itself, and the $L$ and $R$ blocks $\left(\rho_{\text {red }}^{\mathcal{P}_{1}}\right)$ (see Eq. $\left.(11)\right)$ and similarly, the overlap of states in II with itself, and $L$ and $R$ blocks $\left(\rho_{\text {red }}^{\mathcal{P}_{2}}\right)$ (see Eq. (12) for $N-k=1$ and Eq. (13) for $N-k=2$ ).

$$
\begin{aligned}
& \boldsymbol{\rho}_{\text {red }}^{\mathcal{P}_{\mathbf{1}}}=\operatorname{tr}(|\zeta\rangle\langle\zeta|)+\frac{1}{2} \sum_{i=0, j=0}^{\substack{i=1, j=k-2-i}} \mathcal{Z}_{(k-2-i-j)}^{(\mathcal{S}+i)}\left(\frac{1}{2}\right)^{j}|1-i, i\rangle|1\rangle\left\langle i-1, i+1\left|+\frac{1}{2} \sum_{j=0}^{j=k-4} \mathcal{Z}_{(k-4-j)}^{(\mathcal{S}+1)}\left(\frac{1}{2}\right)^{j} \operatorname{tr}(|\overline{2}\rangle\langle\overline{2}|)\right| 1\right\rangle\langle 1| \\
& +\left(\frac{1}{2}\right)^{\substack{k_{6}=k-1, j=k-k_{6}-1}} \sum_{k_{6}=2, j=0}^{(\mathcal{S})} \mathcal{Z}_{\left(k-k_{6}-1-j\right)}\left(\frac{1}{2}\right)^{j} \operatorname{tr}\left(\left|\chi_{k_{6}}\right\rangle\left\langle\chi_{k_{6}}|| 1\right\rangle\left\langle 1|+| \chi_{k_{6}}\right\rangle|1\rangle\left\langle\chi_{k_{6}+1}\right|\right)+\frac{1}{2} \sum_{i=0, j=0}^{j=k-4-i} \mathcal{Z}_{(k-4-(i+j))}^{(\mathcal{S}+1)}\left(\frac{1}{2}\right)^{i+j} \\
& \times \operatorname{tr}\left(| \overline { 2 } \rangle | 1 \rangle \left\langle1|\langle 2|)+\frac{1}{2} \sum_{i=0, j=0, l=0}^{\substack{j=1 \\
l=k-3-(i+j)}} \mathcal{Z}_{(k-3-(i+j+l)}^{(\mathcal{S}+i)}\left(\frac{1}{2}\right)^{j+l} \operatorname{tr}(|1\rangle|1-i, i\rangle|1\rangle\langle\overline{2}|\langle 1-i, i|), \text { where } \mathcal{S}=N-k-1,(11\right.\right. \\
& \boldsymbol{\rho}_{\text {red }}^{\mathcal{P}_{2}}=\left|\gamma_{1}\right\rangle\left\langle\gamma_{1}|+| \gamma_{2}\right\rangle\left\langle\gamma_{2}\right|+\left(\frac{1}{2}\right)^{k-3}\left(\left|\gamma_{1}\right\rangle\left\langle\gamma_{2}|+| \gamma_{2}\right\rangle\left\langle\gamma_{1}\right|\right)+\left[\left(\left|\gamma_{1}\right\rangle+\left|\gamma_{2}\right\rangle\right)\left(\mathcal{D}_{k-2}\langle 1|\langle\overline{2}|\left\langle 1^{\prime}\right|+\mathcal{D}_{k-1}\langle H|\right)+h . c .\right](-1)^{k+1 / 2} \\
& \text { where } N-k=1 \text {, } \\
& \boldsymbol{\rho}_{\text {red }}^{\mathcal{P}_{\mathbf{2}}}=\left(|\xi\rangle\langle\xi|+| \xi\rangle\left\langle\chi_{2}\right|\left\langle 1^{\prime}\right|\right)(-1)^{k / 2-1} \mathcal{D}_{k-1}, \quad \text { where } N-k=2 \text {. }
\end{aligned}
$$

The terms $|\xi\rangle,\left|\gamma_{1}\right\rangle,\left|\gamma_{2}\right\rangle$, and $|\zeta\rangle$, along with an illus- 
the Figs. 2 and 4 . $\mathcal{D}_{k}$ can recursively be generated using $\mathcal{D}_{x}=\mathcal{D}_{x-1}+2 \mathcal{D}_{x-2}$ with the initial condition $\mathcal{D}_{0}=\mathcal{D}_{1}=$ 1. Now if $N-k=1$, periodic states corresponding to the two types, $\mathcal{P}_{1}$ and $\mathcal{P}_{2}$, would overlap with each other and lead to the following additional terms in the expression of the total reduced density matrix of 4-sites given by, $\rho_{\text {red }}^{\mathcal{P}_{12}}=1 / 2^{(k-3)}(-1)^{(k+1) / 2}\left(|\gamma\rangle\left(\left\langle\gamma_{1}\right|+\left\langle\gamma_{2}\right|\right)+\right.$ h.c. $) \mathcal{D}_{k-3}$, where $|\gamma\rangle=|\overline{2}\rangle_{1, N}|N-k-2, k-2\rangle_{2, N-1}$. Hence considering all possible periodic boundary terms, the expression of the reduced density matrix for the system is given by $\rho_{\text {red }}^{\mathcal{P}}=\rho_{\text {red }}^{\mathcal{N} \mathcal{P}}+\rho_{\text {red }}^{\mathcal{P}_{1}}+\rho_{\text {red }}^{\mathcal{P}_{2}}+\rho_{\text {red }}^{\mathcal{P}_{12}}$. Fig. 5 shows the $\mathcal{G}$ of a periodic doped RVB state calculated using the recursion method for upto 300 lattice sites.

The expression of the reduced density matrices obtained using the recursion method can be applied to compute various bipartite as well as multipartite physical quantities that characterize the ground state properties of the system, even for large lattice size. In the following section, we will look at the genuine multipartite entanglement properties of the doped RVB ladder, which can be efficiently obtained using this technique.

\section{GENUINE MULTIPARTY ENTANGLEMENT IN QUANTUM LADDERS}

Here we investigate the multipartite entanglement of a doped quantum spin ladder, under the RVB ansatz. Since the study of GS properties of $t-J$ Hamiltonian is limited to numerical simulations and approximate methods, explicit estimation of multipartite entanglement is extremely difficult for large systems. The doped RVB ansatz for the GS of the $t$ - $J$ model provides a viable alternative to study such quantities. It is known that the RVB liquid state with no holes, $|\Psi\rangle_{N, 0}$, is rotationally invariant under the unitary $U^{\otimes 2 N}$, where $U$ is a local unitary acting on a single qubit ${ }^{19-21}$. In the composite dimer-hole qutrit space, the doped RVB state, $|\Psi\rangle_{k, N-k}$, is invariant under unitary operations of the form $\tilde{U}^{\otimes 2 N}=(U \oplus \mathbb{I})^{\otimes 2 N}$, where $\oplus$ is the direct sum, $\mathbb{I}$ is the scalar 1 and $U$ is an arbitrary single qubit unitary. This invariance property of doped RVB ladders is important in investigating its multipartite entanglement as shown below.

Theorem: The doped RVB ladder state, $|\Psi\rangle_{k, N-k}$, with $2 N$ lattice sites, containing all possible coverings of $k(k \neq 0)$ spin dimers interspersed with $2(N-k)$ holes, is always genuinely multipartite entangled for all ladder topologies that are periodic or infinite along the rails.

Proof: To prove that $|\Psi\rangle_{k, N-k}$ is genuinely multisite entangled, we need to show that the state is entangled across every possible bipartition or alternatively, we have to prove that all reduced density matrices of the system are mixed. Using the invariance of $|\Psi\rangle_{k, N-k}$ under the action of $\tilde{U}^{\otimes 2 N}$, one can show that all $p$-qutrit reduced systems, $\rho^{(p)}=\operatorname{Tr}_{\bar{p}}\left[|\Psi\rangle\left\langle\left.\Psi\right|_{k, N-k}\right]\right.$, obtained by tracing over all but $p(\bar{p})$ sites, is always invariant under $\tilde{U}^{\otimes p}$. Hence, a single qutrit reduced state must have the form, $\rho^{(1)}=p|2\rangle\langle 2|+(1-p) / 2 \mathbb{I}_{2}$, where $\mathbb{I}_{2}=|0\rangle\langle 0|+| 1\rangle\langle 1|$ and $p$ is fixed by the number of holes in the system. The relation shows that $\rho^{(1)}$ is always mixed for $p \neq 1$. Since all $\rho^{(1)}$ are equivalent, the condition $p=1$ is satisfied iff all $2 N$ sites contain holes. Similarly, the nearest neighbor two-site density matrix has the form, $\rho^{(2)}=p_{1}|22\rangle\langle 22|+$ $p_{2} / 9 \mathbb{I}_{9}+p_{3} W_{2}(q)$, where $\mathbb{I}_{9}$ is the identity matrix on $\mathbb{C}^{3} \otimes \mathbb{C}^{3}$ and $W_{2}(q)=q\left|\psi^{-}\right\rangle\left\langle\psi^{-}\right|+(1-q) \mathbb{I}_{4} / 4$ is the Werner state ${ }^{31}$ with $\mathbb{I}_{4}$ being the identity operator on the 4-dimensional space defined in the projected two-qubit spin basis. Now, $\rho^{(2)}$ is pure when $p_{1}=p_{2}=0$ and $q=1$. Which implies that it is pure iff the entire lattice is either filled with holes or is a single dimer covering, and these options are disallowed by the premise. Therefore, $\rho^{(1)}$ and $\rho^{(2)}$ are always mixed and $|\Psi\rangle_{k, N-k}$ is always entangled across these bipartitions.

However, we want to show that all possible bipartitions, irrespective of the number of sites, are always mixed. To prove this let us assume now that an arbitrary $p$-site density matrix $\left(\rho^{(p)}\right)$ is pure, which implies that $|\Psi\rangle_{k, N-k}$ is separable along that $p: 2 N-p$. Let $p=p_{1}+j$, where $j=1$ or 2 such that $|j|<\left|p_{1}\right|(|\cdot|$ is the cardinality of the argument). For the periodic or infinite ladder, one can always find another equivalent pure density matrix, $\rho^{(q)}$, such that $q=q_{1}+j$ and $|p|=|q|$, where $j$-sites overlap. By assumption, both $\rho^{(p)}$ and $\rho^{(q)}$ are pure. Using strong subadditivity of von Neumann entropy, $S(\sigma)=-\operatorname{tr}\left(\sigma \log _{2} \sigma\right)^{32}$, we obtain $S\left(\rho^{\left(p_{1}\right)}\right)+S\left(\rho^{\left(q_{1}\right)}\right) \leq S\left(\rho^{\left(p_{1}+j\right)}\right)+S\left(\rho^{\left(q_{1}+j\right)}\right)$. Now $S\left(\rho^{\left(p_{1}+j\right)}\right)=S\left(\rho^{\left(q_{1}+j\right)}\right)=0$, since $\rho^{(p)}$ and $\rho^{(q)}$ are pure. Since $S \geq 0$, we have $S\left(\rho^{\left(p_{1}\right)}\right)=S\left(\rho^{\left(q_{1}\right)}\right)=0$, and therefore $S\left(\rho^{(j)}\right)=0$ implying $\rho^{(j)}$ is pure, which is not true since all $\rho^{(1)}$ and $\rho^{(2)}$ are mixed under finite doping. The contradiction implies that all reduced density matrices, $\rho^{(p)}$ are mixed and all $p: 2 N-p$ are entangled.

We note that the above proof does not include the $p$ : $2 N-p$ bipartitions where no equivalent $\rho^{(q)}$ with overlap is feasible, such as the bipartition between the two legs of the ladder. However, in such cases, the theorem can be proved using a different argument. We assume that the legs, $L_{i}$ and $L_{i}^{\prime}$ of $|\Psi\rangle_{k, N-k}$ are pure and thus the entire state is separable along that $N: N$. For the above condition to be satisfied, all reduced states along the rungs, $\rho_{\left(L_{k}, L_{k}^{\prime}\right)}^{(2)}, \forall k$, must be separable. However, as can be shown by using recursive method, such nearest-neighbor $\rho^{(2)}$ states are always entangled. Hence, the doped RVB state is genuinely multipartite entangled.

Let us now quantify the genuine multipartite entanglement in doped RVB ladders and characterize its variation with the electron density. Towards that aim, one needs to find a computable measure of genuine multiparty entanglement, which in our work is the "generalized geometric measure" $(\mathrm{GGM})^{22}\left(\mathrm{cf}^{23}\right)$. In the forthcoming section, we provide all the necessary details required to compute GGM for any arbitrary $N$-party pure quantum state. 


\section{GEUINE MULTISITE ENTANGLEMENT MEASURE}

The GGM, $\mathcal{G}$, of an N -party pure quantum state $|\phi\rangle$ is a computable measure and is basically the optimized fidelity distance of the state from the set of all states that are not genuinely multiparty entangled. In particular, the GGM $\mathcal{G}(|\phi\rangle)$ can be evaluated as

$$
\mathcal{G}(|\phi\rangle)=1-\lambda_{\max }^{2}
$$

where $\lambda_{\max }=\max \left|\left\langle\xi_{n} \mid \phi\right\rangle\right|,\left|\xi_{n}\right\rangle$ is an $N$-party nongenuinely multisite entangled quantum state and the maximization is performed over the set of all such states. For pure quantum states, it was shown that GGM can be effectively computed using the straightforward relation ${ }^{22}$

$$
\begin{gathered}
\mathcal{G}(|\phi\rangle)=1-\max \left\{\lambda_{A: B}^{2} \mid A \cup B=A_{1}, \ldots, A_{N},\right. \\
A \cap B=\phi\},
\end{gathered}
$$

where $\lambda_{A: B}$ is the maximum Schmidt coefficient in all possible bipartion split of $A: B$ of the given state $|\phi\rangle$.

Genuine multipartite entanglement is a well understood physical property in entanglement theory (see Ref. ${ }^{33}$ ), which essentially captures the presence of entanglement between every constituent of a many-body system. In contrast, measures such as entanglement entropy and entanglement of formation are essentially bipartite entanglement measure, which do not necessarily say anything about the global entanglement properties of a many-body state. Although entanglement entropy is important in studying cooperative phenomena such as area laws, it is not adequate to study the multipartite entanglement properties of many-body systems. Presence of multipartite entanglement may give rise to interesting cooperative properties that are not necessarily exhibited by restricting to bipartite entanglement. An obvious advantage of using GGM, is that it can be efficiently calculated through the reduced density matrices of a many-body quantum state. One should stress here that entanglement entropy captures the distribution of entanglement between two blocks of the system constituted of connected cluster of spins. In comparison, GGM allows us to characterize the entanglement between all possible partitions of the system into two, three, four, ... blocks, comprising of connected as well as disconnected group of spins, which provides insight about the cooperative properties of the ground state, beyond correlation decay or area laws ${ }^{34}$.

\section{TRENDS OF GENUINE MULTISITE ENTANGLEMENT: GS OF T-J LADDER VS DOPED RVB STATE}

We now consider a quantum spin-1/2 ladder model, consisting of an arbitrary numbers of holes and spin particles, and consider the short-ranged RVB state as a framework to study its GS multiparty entanglement properties.
The model can be derived using second order perturbation theory from the Hubbard model in the limit of large on-site interaction. ${ }^{1-4}$ The $t-J$ Hamiltonian on a ladder can be written as

$$
\mathcal{H}=-t \sum_{\langle i, j\rangle, \sigma} \mathcal{P}_{G}\left(c_{i \sigma}^{\dagger} c_{j \sigma}+\text { h.c. }\right) \mathcal{P}_{G}+J \sum_{\langle i, j\rangle} \vec{S}_{i} \cdot \vec{S}_{j}
$$

where $c_{i \sigma}\left(c_{i \sigma}^{\dagger}\right)$ is the fermionic annihilation (creation) operator of spin $\sigma(=\{\uparrow, \downarrow\})$, and $\vec{S}_{i}$ is the triad of spin$1 / 2$ operators, at site $i$. The Heisenberg exchange coupling $(J)$ is isotropic along the rungs and legs while $t$ represents the transfer energy and the expression $\langle i, j\rangle$ denotes that the sum is taken over nearest neighbor (NN) sites. $\mathcal{P}_{G}$ is the Gutzwiller projector $\Pi_{i}\left(1-n_{i \uparrow} n_{i \downarrow}\right)$ which enforces at most single occupancy at each lattice site. This ensures that the undoped state physically represents a Mott insulator. The $t-J$ model, under finite doping, exhibits a rich phase diagram ${ }^{8-12}$. Note that these models can potentially be realized in fermionic ultracold gases at high energy scales ${ }^{35}$. For moderate sized $t$ - $J$ ladders, at half-filling, the Hamiltonian in Eq. (16) can be exactly diagonalized, provided certain properties of the system are invoked. For example, the spin number Hamiltonian, $\hat{N}=\sum_{i}(|0\rangle\langle 0|+| 1\rangle\langle 1|)_{i}$, and the total spin along the z-axis, $\hat{S}^{z}=\sum_{i} S_{i}^{z}$ commute with the Hamiltonian, $\mathcal{H}$. Hence, the Hamiltonian can be block-diagonalized in the $\left(\mathbb{C}^{3}\right)^{\otimes 2 N}$ Hilbert space basis for different total spin $\hat{S}^{z}$ and electron density $n_{e l}=\langle\hat{N}\rangle / 2 N$. For our case, we assume that the spins form an initial insulating phase with $\hat{S}^{z}=0$, and with $n_{e l}$ varying from 0 to 1 . Note that $n_{e l}$ $=0$ and 1 , correspond to a completely vacant and occupied lattice, respectively. For $n_{e l}=1$, the state is an insulating RVB spin liquid. The doping concentration is denoted by $x=1-n_{e l}$. In our work, we have developed a numerical algorithm ${ }^{36}$, based on the Lanczos $\operatorname{method}^{37}$, to exactly solve the composite hole-dimer qutrit system. By dividing the Hilbert space in different subspaces, according to the hole concentration $x$ and total $\hat{S}^{z}$, exact ground state of the $t$ - $J$ Hamiltonian can be obtained for upto 14 qutrits, with even number of holes.

Although we have shown that the doped RVB state is always genuinely multiparty entangled, a quantitative analysis of $\mathcal{G}$ requires its computation for large systems. Using the analytical recursion method proposed in the work, one can recursively build the doped RVB state and subsequently obtain its relevant reduced density matrices which is necessary to estimate $\mathcal{G}$. Figure 5 shows the behavior of the $\mathcal{G}$ with increasing $n_{e l}$. We observe that at $n_{e l}=0, \mathcal{G}$ vanishes as expected since it corresponds to a product state, containing only holes. The maximum $\mathcal{G}$ is achieved at a critical density, $n_{e l}=n_{c} \approx 0.56$. Interestingly, we find that this critical value of $n_{c}$ with respect to $\mathcal{G}$ corresponds to that of the superconducting phase of the $t$ - $J$ model.

We now consider the behavior of genuine multisite entanglement in the GS of the periodic $t-J$ ladder, obtained through exact diagonalization. Figure 6 , shows the varia- 


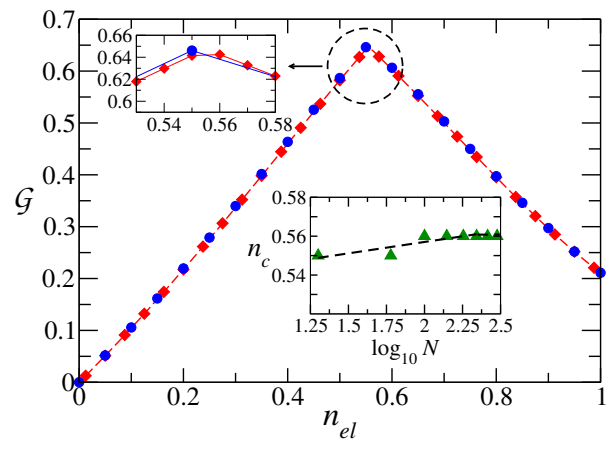

FIG. 5. (Color online.) Genuine multisite entanglement in doped RVB ladder. Variation of $\mathcal{G}$ with $n_{e l}$ in doped RVB ladder states, for $2 N=40$ (blue circles) and 200 (red diamonds) lattice sites. The top inset magnifies the encircled region in the plot. The bottom inset shows the scaling of $n_{c}$ with the $\log _{10} N$. The inset shows that as $N$ increases (plotted up to 300 sites), $n_{c}$ converges to 0.56 . This is to be compared to the result for the systems described by the $t-J$ model in the superconducting regime, in Fig. 6.

tion of $\mathcal{G}$ with $n_{e l}$ for different moderately-sized systems. We observe that the behavior of $\mathcal{G}$ is qualitatively equivalent to those corresponding to doped RVB states (see Fig. 5). Below a certain critical density $\left(n_{c}\right)$, i.e., in the region where $n_{e l}<n_{c}, \mathcal{G}$ scales linearly with $n_{e l}$, independent of $J / t$. This is due to the fact that $\mathcal{G}$ is obtained from the 1:rest bipartition, where the single-site density matrix is diagonal in the computational basis, with elements $\left[1-n_{e l}, n_{e l} / 2, n_{e l} / 2\right]$. When $1 \geq n_{e l} \geq n_{c}$, the $\mathcal{G}$ is a function of both $n_{e l}$ and $J / t$. For the $t-J$ ladders, the maximum $\mathcal{G}$ is achieved at a critical density, $n_{c} \approx 0.65$ which is close to that obtained using the doped RVB state ansatz. However, the small discrepancy in the exact values of the electron densities need to account for finite size effect. Moreover, we infer that this critical density mark the onset of a superconducting phase in the two-legged $t-J$ ladder. For example in $t$ - $J$ ladder with $J / t \approx 0.6$, the superconducting phase has been predicted to occur for relatively high values of $n_{e l}{ }^{9}$, which is close to the critical density corresponding to the $\mathcal{G}$, as obtained from our analyses.

Even though the microscopic theory behind high- $T_{c}$ superconductivity $^{13,14,38-40}$ remains unresolved ${ }^{41,42}, t-J$ ladder stands out as an important framework for understanding this novel phenomena ${ }^{43,44}$. Furthermore, the short-range RVB ansatz has been pitched to describe the superconducting states of the $t-J$ ladder ${ }^{15,16}$. The RVB state is a possible GS of the half-filled $t-J$ ladder ${ }^{45}$ and, upon finite doping, provides a simple mechanism to describe high- $T_{c}$ superconductivity. In this respect, our work indicates that $\mathcal{G}$ bears the signature of the $t$ - $J$ ladder entering into the superconducting phase and even the minimalistically designed doped RVB state considered in this work supports this feature, at least at the level of multiparty entanglement. Based on the behavior of both doped RVB states and exact GS of the $t$ - $J$ Hamiltonian, one can hypothesize that the trend of $\mathcal{G}$ can detect the superconducting phase boundary, irrespective of the size of the ladder. Note, however that we do not claim to detect a high $T_{c}$ superconducting phase by using the genuine multipartite entanglement as an order parameter. This is also not the primary motivation of our work, which is to construct an efficient recursive method for evaluating bipartite as well as multiparty observables in large doped RVB states. As an useful spin-off to our main results, we are able to show that for some parameter ranges, $\mathcal{G}$ may serve as an indicator to whether the system has entered into the SC phase or not. It is plausible that one would require further physical properties along with the GGM to identify all the phases. Since there exists, as yet, no order parameter that can uniquely identify all the relevant phases of the ground states of doped Hubbard or $t-J$ model $^{46}$, the applicability of $\mathcal{G}$ as a suitable order parameter requires further investigation.

An important point in our work is the use of a nonvariational RVB state as the GS of the doped quantum spin ladder. It is clear that a variational RVB (vRVB) state, which lends possible support to $d$-wave pairing, is a more suitable state to study the doped ladder. However, vRVB states, in general, do not possess a recursive form that allows computation of reduced states with high efficiency in large systems, as the number of parameters to optimize increases exponentially. However, our results show that by omitting the variation in the coefficients of the covering required to build the RVB state, we obtain a significant advantage in computation power, which allows us to compute $\mathcal{G}$ in large doped ladders. Comparison with exact GS of the $t-J$ ladder shows that the non-variational RVB state, quite accurately simulates the behavior of $\mathcal{G}$. But we do think that efficient recursions for the certain variational RVB states is an important problem for future tasks.

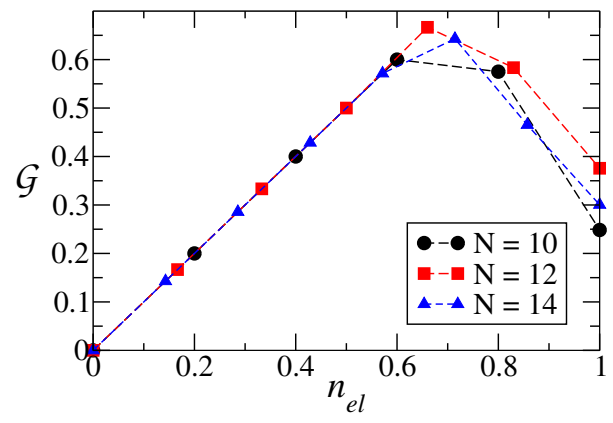

FIG. 6. (Color online). Genuine multisite entanglement in the $t$ - $J$ ladder. Variation of $\mathcal{G}$ with $n_{e l}$ for the exact GS of the $t$ - $J$ ladder Hamiltonian, given in Eq. (16), for $N=10$, 12 , and 14 . $\mathcal{G}$ reaches its maximum value at $n_{c} \approx 0.65$. Here $J / t=0.66$. All quantities plotted are dimensionless.

\section{CONCLUSIONS}

In this paper, we adopted two techniques for studying multisite entanglement in doped quantum spin lad- 
ders. Firstly, we consider the doped RVB states as possible ground states of the $t-J$ Hamiltonian, which we have shown to be always genuinely multiparty entangled. To overcome the limitations of exact diagonalization, we introduced a recursion method to generate the doped RVB state and to compute its reduced density matrices. By using the iterative method, we find that we can compute the genuine multiparty entanglement of doped RVB ladders, for large systems under finite doping of the ladder. We found that the maximum value occurs at doping concentration $n_{e l}=0.56$. Secondly, we use an exact diagonalization method for the $t-J$ Hamiltonian, for upto 14 sites and observe that the GS of the Hamiltonian is also genuinely multipartite entangled, with maximum entanglement occurring at the superconducting phase boundary, where the electron density $n_{e l} \approx 0.65$.

We note that the primary outcome of our work is an analytical recursion method to evaluate the genuine multipartite entanglement in RVB ladders with finite doping. An immediate offshoot of our results is the connection between maximal entanglement in doped RVB states and the high- $T_{c}$ superconducting phases of the $t-J$ Hamiltonian. In this regard, we would like to mention that even though GGM may apparently be useful in signalling the onset of the high- $T_{c}$ superconducting phase, it is possible that the different phases of the Hubbard or the $t-J$ model can not be completely characterized by just using entanglement. Recently, using the behavior of multiparty entanglement, attempts have been made to get more accurate insight about the phase boundaries which emerge in the ground state configuration of $X X Z$ quantum spin ladders ${ }^{47,48}$. However, it has been shown that there are regions in the parameter space at which multiparty entanglement alone fails to a provide a conclusive phase diagram and one needs to study the behavior of other ground state properties such as magnetization and spin correlation functions in order to obtain a complete picture of the different phase boundaries ${ }^{48}$. In a similar vein, for the case of doped ladders, it is plausible that one would require further physical properties along with the GGM, to characterize different phases of the $t-J$ Hamiltonian. This requires further investigations on the model which are planned in forthcoming works. Apart from this, there has also been attempt to quantify the bipartite entanglement of certain multipartite pure states, like the Bardeen-Cooper-Schrieffer (BCS) state of superconducting compounds ${ }^{49}$ which shed light on the relation of entanglement to that of the superconducting order parameter. We believe that the extension of such investigations to the case of high- $T_{c}$ cuprates may uncover interesting underlying microscopic properties.

\section{ACKNOWLEDGEMENT}

HSD acknowledges funding by the Austrian Science Fund (FWF), project no. M 2022-N27, under the Lise Meitner programme of the FWF.
${ }^{1}$ K. A. Chao, J. Spałek, and A. M. Oleś, Kinetic exchange interaction in a narrow S-band, J. Phys. C 10, L271 (1977); Canonical perturbation expansion of the Hubbard model, Phys. Rev. B 18, 3453 (1978); A. Auerbach, Interacting Electrons and Quantum Magnetism, Springer, Berlin, 1998.

${ }^{2}$ F. C. Zhang and T. M. Rice, Effective Hamiltonian for the superconducting $\mathrm{Cu}$ oxides, Phys. Rev. B 37, 3759(R) (1988).

3 V. J. Emery and G. Reiter, Quasiparticles in the copperoxygen planes of high- $T_{c}$ superconductors: An exact solution for a ferromagnetic background, Phys. Rev. B 38, 11938(R) (1988).

4 Another important derivation of the $t$ - $J$ model was obtained by Zhang and Rice from the three-band Hubbard model that describe the cuprate planes in high- $T_{c}$ superconductors $^{2}\left(\right.$ cf. $\left.^{3}\right)$. The $t$ - $J$ model together with the one-band and three-band Hubbard models, in various limits, effectively describe the Hamiltonians for the study of high- $T_{c}$ superconductivity, although disagreements exist $^{13,14}$

${ }^{5}$ E. Dagotto, J. Riera, and D. Scalapino, Superconductivity in ladders and coupled planes, Phys. Rev. B 45, 5744(R) (1992); S. Gopalan, T. M. Rice, and M. Sigrist, Spin ladders with spin gaps: A description of a class of cuprates, Phys. Rev. B 49, 8901 (1994); T. Barnes and J. Riera, Susceptibility and excitation spectrum of $(\mathrm{VO})_{2} \mathrm{P}_{2} \mathrm{O}_{7}$ in ladder and dimer-chain models, Phys. Rev. B 50, 6817 (1994).
${ }^{6}$ M. Sigrist, T. M. Rice, and F. C. Zhang, Superconductivity in a quasi-one-dimensional spin liquid, Phys. Rev. B 49, 12058 (1994); R. M. Noack, S. R. White, and D. J. Scalapino, Correlations in a Two-Chain Hubbard Model, Phys. Rev. Lett. 73, 882 (1994); D. V. Khveshchenko and T. M. Rice, Spin-gap fixed points in the double-chain problem, Phys. Rev. B 50, 252 (1994); B. Edegger, V. N. Muthukumar, and C. Gros, Gutzwiller-RVB theory of high-temperature superconductivity: Results from renormalized mean-field theory and variational Monte Carlo calculations, Adv. Phys. 927, 1033 (2007).

7 M. Ogata, M. U. Luchini, and T. M. Rice, Spin gap in a generalized one-dimensional $t-J$ model, Phys. Rev. B 44, 12083(R) (1991); M. Imada, Spin-gap state and superconducting correlations in a one-dimensional dimerized $t$-J model, Phys. Rev. B 48, 550 (1993), and references therein.

8 M. Ogata, M. Luchini, S. Sorella, and F. Assaad, Phase diagram of the one-dimensional $t$-J model, Phys. Rev. Lett 66, 2388 (1991); S. R. White and D. J. Scalapino, Hole and pair structures in the $t-J$ model, Phys. Rev. B 55, 6504 (1997); S. R. White and D. J. Scalapino, Density Matrix Renormalization Group Study of the Striped Phase in the 2D t-J Model, Phys. Rev. Lett. 80, 1272 (1998); A. Moreno, A. Muramatsu, and S. R. Manmana, Ground-state phase diagram of the one-dimensional $t$ - $J$ model, Phys. Rev. B 83, 205113 (2011); S. Guertler and H. Monien, Unveiling the Physics of the Doped Phase of the $t J$ Model on 
the Kagome Lattice, Phys. Rev. Lett. 111, 097204 (2013).

9 K. Sano, Possible Phase Diagram of a $t$-J Ladder Model, J. Phys. Soc. Jpn. 65, 1146 (1996).

10 M. Troyer, H. Tsunetsugu, and T. M. Rice, Properties of lightly doped t-J two-leg ladders, Phys. Rev. B 53, 251 (1996).

11 G. Sierra, M. A. Martín-Delgado, J. Dukelsky, S. R. White, and D. J. Scalapino, Dimer-hole-RVB state of the two-leg $t-J$ ladder: A recurrent variational ansatz, Phys. Rev. B 57, 11666 (1998).

12 F. D. M. Haldane, 'Luttinger liquid theory' of onedimensional quantum fluids. I. Properties of the Luttinger model and their extension to the general $1 D$ interacting spinless Fermi gas, J. Phys. C: Solid State Phys. 14, 2585 (1981); N. Nagaosa and P. A. Lee, Normal-state properties of the uniform resonating-valence-bond state, Phys. Rev. Lett. 64, 2450 (1990); C. A. Hayward and D. Poilblanc, Luttinger-liquid behavior and superconducting correlations in $t$-J ladders, Phys. Rev. B 53, 11721 (1996).

13 E. Dagotto, Correlated electrons in high-temperature superconductors, Rev. Mod. Phys. 66, 763 (1994).

14 P. A. Lee, N. Nagaosa, and X.-G. Wen, Doping a Mott insulator: Physics of high-temperature superconductivity, Rev. Mod. Phys. 78, 17 (2006).

${ }^{15} \mathrm{P} . \mathrm{W}$. Anderson, Resonating valence bonds: A new kind of insulator?, Mat. Res. Bull. 8, 153 (1973); P. Fazekas and $\mathrm{P} . \mathrm{W}$. Anderson, On the ground state properties of the anisotropic triangular antiferromagnet, Philos. Mag. 30, 23 (1974).

16 G. Baskaran, Z. Zou. P. W. Anderson, The resonating valence bond state and high- $T_{c}$ superconductivity-A mean field theory, Solid state communications 63, 973 (1987); P. W Anderson, G Baskaran, Z Zou, and T Hsu, Resonatingvalence-bond theory of phase transitions and superconductivity in $\mathrm{La}_{2} \mathrm{CuO}$-based compounds, Phy. Rev. lett. 58, 2790 (1987); G. Baskaran and P. W. Anderson, Gauge theory of high-temperature superconductors and strongly correlated Fermi systems, Phys. Rev. B 37, 580(R) (1988); G. Baskaran, Resonating Valence Bond States in 2 and 3D: Brief History and Recent Examples, Indian J. Phys. 89, 583 (2006).

17 A. Osterloh, L. Amico, G. Falci, and R. Fazio, Scaling of Entanglement close to a Quantum Phase Transitions, Nature 416, 608 (2002).

18 M. Lewenstein, A. Sanpera, V. Ahufinger, B. Damski, A. Sen(De), and U. Sen, Adv. Phys. 56, 243-379 (2007).

19 A. Chandran, D. Kaszlikowski, A. Sen(De), U. Sen, and V. Vedral, Regional Versus Global Entanglement in Resonating-Valence-Bond States, Phys. Rev. Lett. 99, 170502 (2007).

${ }^{20}$ H. S. Dhar and A. Sen(De), Entanglement in resonating valence bond states: ladder versus isotropic lattices, J. Phys. A: Math. Theor. 44, 465302 (2011).

${ }^{21}$ H. S. Dhar, A. Sen(De), and U. Sen, Characterizing Genuine Multisite Entanglement in Isotropic Spin Lattices, Phys. Rev. Lett. 111, 070501 (2013).

22 A. Sen(De) and U. Sen, Channel capacities versus entanglement measures in multiparty quantum states, Phys. Rev. A 81, 012308 (2010); A. Sen(De) and U. Sen, Bound Genuine Multisite Entanglement: Detector of Gapless-Gapped Quantum Transitions in Frustrated Systems, arXiv:1002.1253 [quant-ph](2010).

23 A. Shimony, Degree of entanglement, Ann. NY Acad. Sci.755, 675 (1995); H. Barnum and N. Linden, Mono- tones and invariants for multi-particle quantum states, J. Phys. A 34, 6787 (2001); M. Blasone, F. DellAnno, S. De Siena, and F. Illuminati, Hierarchies of geometric entanglement, Phys. Rev. A 77, 062304 (2008).

24 T.-C. Wei, D. Das, S. Mukhopadyay, S. Vishveshwara, and P. M. Goldbart, Global entanglement and quantum criticality in spin chains, Phys. Rev. A 71, 060305(R) (2005); T. R. de Oliveira, G. Rigolin, M. C. de Oliveira, and E. Miranda, Multipartite Entanglement Signature of Quantum Phase Transitions, Phys. Rev. Lett. 97, 170401 (2006); R. Orús, Universal Geometric Entanglement Close to Quantum Phase Transitions, Phys. Rev. Lett. 100, 130502 (2008); M. Hofmann, A. Osterloh, and O. Gühne, Scaling of genuine multiparticle entanglement close to a quantum phase transition, Phys. Rev. B 89, 134101 (2014); A. Biswas, R. Prabhu, A. Sen(De), and U. Sen, Genuinemultipartite-entanglement trends in gapless-to-gapped transitions of quantum spin systems, Phys. Rev. A 90, 032301 (2014).

25 R. Ramanathan, D. Kaszlikowski, M. Wiesniak, and V. Vedral, Entanglement in doped resonating valence bond states, Phys. Rev. B 78, 224513 (2008).

26 The counting of all possible dimer coverings with holes on a ladder configuration is not easily tractable using conventional exact methods. In fact, in a 2D quantum spin lattice, the arrangement of monomer and dimer coverings, as encountered in the doped RVB system, is $N P$-complete ${ }^{25}$. Also see, M. Jerrum, J. Stat. Phys. 48, 121 (1987).

27 M. Roncaglia, G. Sierra, and M. A. Martin-Delgado, Dimer resonating valence bond state of the four-leg Heisenberg ladder: Interference among resonances, Phys. Rev. B 60, 12134 (1999)

${ }^{28}$ H. S. Dhar, A. Sen(De), and U. Sen, The density matrix recursion method: genuine multisite entanglement distinguishes odd from even quantum spin ladder states, New J. Phys. 15, 013043 (2013); S. S. Roy, H. S. Dhar, D. Rakshit, A. Sen(De), and U. Sen, Diverging scaling with converging multisite entanglement in odd and even quantum Heisenberg ladders, New J. Phys. 18, 023025 (2016).

29 Y. Fan and M. Ma, Generating-function approach to the resonating-bond state on the triangular and square ladders, Phys. Rev. B 37, 1820 (1988).

30 G. Sierra and M. A. Martín-Delgado, Short-range resonating-valence-bond state of even-spin ladders: A recurrent variational approach, Phys. Rev. B 56, 8774 (1997).

31 R. F. Werner, Quantum states with Einstein-PodolskyRosen correlations admitting a hidden-variable model. Phys. Rev. A 40, 4277 (1989).

32 E. H. Lieb and M. B. Ruskai, Proof of the strong subadditivity of quantum-mechanical entropy, J. Math. Phys. (N.Y.) 14, 1938 (1973).

33 R. Horodecki, P. Horodecki, M. Horodecki, and K. Horodecki, Quantum entanglement, Rev. Mod. Phys. 81, 865 (2009).

34 T.-C. Wei, D. Das, S. Mukhopadyay, S. Vishveshwara, and P. M. Goldbart, Global entanglement and quantum criticality in spin chains, Phys. Rev. A 71, 060305(R) (2005); X.-F. Qian, T. Shi, Y. Li, Z. Song, and C.-P. Sun, Characterizing entanglement by momentum jump in the frustrated Heisenberg ring at a quantum phase transition, Phys. Rev. A 72, 012333 (2005); T. R. de Oliveira, G. Rigolin, M. C. de Oliveira, and E. Miranda, Multipartite Entanglement Signature of Quantum Phase Transitions, Phys. Rev. Lett. 
97, 170401 (2006); R. Orús, Universal geometric entanglement close to quantum phase transitions, Phys. Rev.Lett. 100, 130502 (2008); R. Orús, S. Dusuel, and J. Vidal, Equivalence of critical scaling laws for many-body entanglement in the Lipkin-Meshkov-Glick model, ibid. 101, 025701 (2008); R. Orús and T.-C. Wei, Visualizing elusive phase transitions with geometric entanglement, Phys. Rev. B 82, 155120 (2010).

35 A. Eckardt and M. Lewenstein, Controlled hole doping of a Mott insulator of ultracold fermionic atoms, Phys. Rev. A 82, 011606(R) (2010).

${ }^{36}$ We develop a numerical algorithm, based on the Lanczos $\operatorname{method}^{37}$, to exactly solve the composite hole-dimer qutrit system. By dividing the Hilbert space in different subspaces, according to the hole concentration and total $S_{z}$, exact ground state of the $t-J$ Hamiltonian can be obtained for moderate system size. The algorithm is implemented using codes written in MatLab and Fortran90. Also, see the Appendix for more details.

37 C. Lanczos, An Iteration Method for the Solution of the Eigenvalue Problem of Linear Differential and Integral Operators, J. Res. Natl. Bur. Stand. 45, 255 (1950); H. Nishimori, TitpackNumerical diagonalization routines and quantum spin Hamiltonians, AIP Conf. Proc. 248, 269 (1991).

38 J. G. Bednorz, and K. A. Müller, 1986, Possible high Tc superconductivity in the $\mathrm{Ba}-\mathrm{La}-\mathrm{Cu}-\mathrm{O}$ system, Z. Phys. B 64, 189 (1986).

39 P. W. Anderson, The Theory of Superconductivity in the High-Tc Cuprate Superconductors, Princeton University Press, Princeton (1997); P. Anderson, P. Lee, M. Randeria, T. M. Rice, N. Trivedi, F. Zhang, The physics behind high- temperature superconducting cuprates: the 'plain vanilla' version of RVB, J. Phys. Condens. Matter 16, 755 (2004).

40 B. Keimer, S. A. Kivelson, M. R. Norman, S. Uchida, and J. Zaanen, From quantum matter to high-temperature superconductivity in copper oxides, Nature (London) 518, 179 (2015).

41 N. F. Mott., The Basis of the Electron Theory of Metals, with Special Reference to the Transition Metals, Proc. Phys. Soc. A 62, 416 (1949).

42 A. J. Leggett, What DO we know about high Tc?, Nat. Phys. 2, 134 (2006); A. Mann, High-temperature superconductivity at 25: Still in suspense, Nature (London) 475, 280 (2011).

43 P. W. Anderson, The Resonating Valence Bond State in $\mathrm{La}_{2} \mathrm{CuO}_{4}$ and Superconductivity, Science 235, 1196 (1987).

44 C. Gros, Superconductivity in correlated wave functions, Phys. Rev. B 38, 931(R) (1988).

45 S. R. White, R. M. Noack, and D. J. Scalapino, Resonating Valence Bond Theory of Coupled Heisenberg Chains, Phys. Rev. Lett. 73, 886 (1994).

46 S. Sachdev, Quantum phases and phase transitions of Mott insulators, Lect. Notes Phys. 645, 381 (2004).

47 Y. Chen, P. Zanardi, Z. D. Wang, and F. C. Zhang, Sublattice entanglement and quantum phase transitions in antiferromagnetic spin chains, New J. Phys. 8, 97 (2006).

48 S. S. Roy, H. S. Dhar, D. Rakshit, A. Sen(De), and U. Sen, Detecting phase boundaries of quantum spin-1/2 XXZ ladder via bipartite and multipartite entanglement transitions, arXiv:1612.06831 [quant-ph](2016).

49 M. A. Martín-Delgado, Entanglement and Concurrence in the BCS State, arXiv:0207026 [quant-ph](2002). 